A Massive Star Odyssey, from Main Sequence to Supernova

Proceedings IAU Symposium No. 212, (C) 2003 IAU

K.A. van der Hucht, A. Herrero \& C. Esteban, eds.

\title{
Line-blanketing effects in atmospheres of O-type stars
}

\author{
Fabrice Martins and Daniel Schaerer \\ Laboratoire d'Astrophysique, Observatoire Midi-Pyrénées, \\ 14 Avenue Edouard Belin, F-31400 Toulouse, la France
}

\begin{abstract}
Line-blanketing effects in atmospheres of O-type stars are studied based on models computed with the non-LTE spherically expanding code CMFGEN (Hillier \& Miller 1998). We show that the inclusion of metals leads to a reduction of the effective temperature scale by $\sim 1500 \mathrm{~K}$ at spectral type 09.5 , up to $\sim 4000 \mathrm{~K}$ at spectral type 03 . This change of the $T_{\text {eff }}$ scale implies a reduction of the luminosity by $\sim 0.1 \mathrm{dex}$ and a decrease of the ionising flux by $\sim 50 \%$ for a given spectral type.
\end{abstract}

\section{Introduction}

The inclusion of metals in model atmospheres of O-type stars (line-blanketing effect) is a complex task. Various attempts have been made since the work of Abbott \& Hummer (1985), e.g., by Schaerer \& Schmutz (1994), Schmutz (1998), Hubeny et al. (1998), and Herrero et al. (2000). Hillier \& Miller (1998) with a super-level approach and Pauldrach et al. (2001) with an opacity sampling method made significant improvements. We show here the results of a quantitative study of line-blanketing effects in O-type dwarf models computed with the code CMFGEN (Hillier \& Miller 1998). A full account of our study can be found in Martins et al. (2002).

\section{Results}

The inclusion of metals in model atmospheres of O-type dwarfs leads to a modification of both the atmospheric structure and the emergent spectrum. In the inner part, photons are backscattered towards the inner atmosphere so that, in order to fullfill the flux, the temperature rises (Figure 1) which increases the ionisation, and hence modifies the strength of the He classification lines. This leads to a cooler $T_{\text {eff }}$ scale compared to previous calibrations based on $\mathrm{H}-\mathrm{He}$ analysis (Figure 1). As a consequence, the luminosity is also reduced by $\sim 0.1$ dex. In the outer part of the atmosphere, the blocking of the EUV flux by metal opacities leads to a reduction of the ionisation, and escape of photons through metallic lines decreases the temperature (see Martins et al. 2002 for details).

Another important effect of line blanketing is the reduction of ionising fluxes. This is due to two effects: $(i)$ the inclusion of new bound-free opacities from metals blocks the EUV flux; and (ii) the cooler $T_{\text {eff }}$ scale implies a smaller number of ionising photons for a given spectral type. For dwarfs stars, 

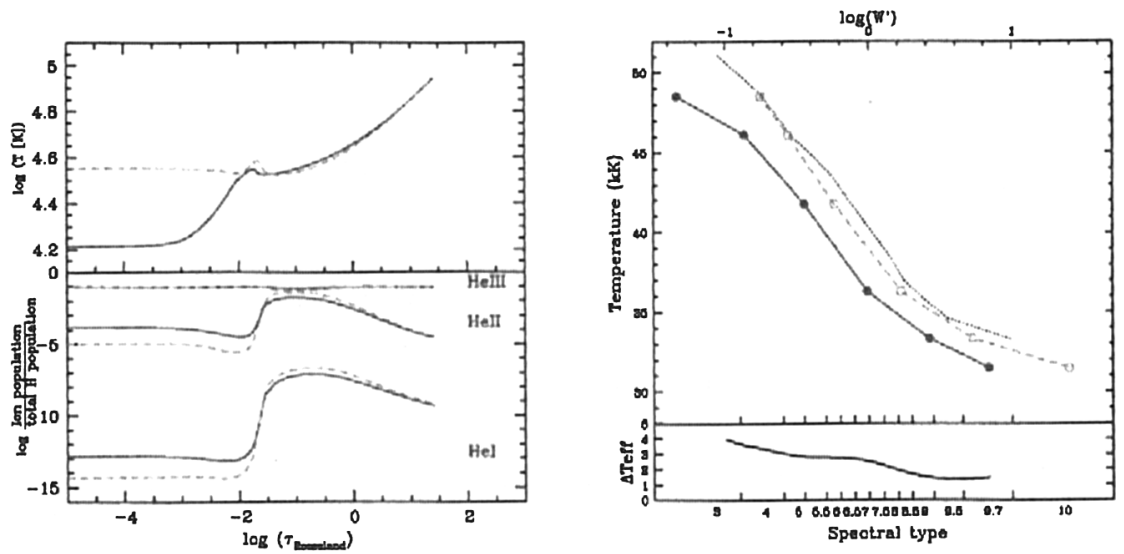

Figure 1. Temperature and He-ionisation structure (left) and $T_{\text {eff }}$ scale (right). Solid line is for line-blanketed models; dashed line is for pure $\mathrm{H}-\mathrm{He}$ models. The $T_{\text {eff }}$ scale of Vacca et al. (1996) is also shown by a dotted line.

a reduction of $\sim 50 \%$ of the ionising flux is found. This has important consequences for the study of $\mathrm{H}$ II regions.

\section{Conclusion}

The reduction of the $T_{\text {eff }}$ scale due to line blanketing predicted by different authors (Abbott \& Hummer 1985; Schaerer \& Schmutz 1994) has been studied quantitatively for O-type dwarfs. Our results are in agreement with recent analyses of individual stars, e.g., by Hubeny et al. (1998), Fullerton et al. (2000), Crowther et al. (2002), and Herrero et al. (2002).

\section{References}

Abbott, D.C., Hummer, D.G. 1985, ApJ 294, 286

Crowther, P.A., Hillier, D.J., Evans, C.J., et al. 2002, ApJ 579, 774

Fullerton, A.W., Crowther, P.A., De Marco, O., et al. 2000, ApJ (Letters) 538, L43

Herrero, A., Puls, J., Najarro, F. 2002, A\&A 396, 949

Herrero, A., Puls, J., Villamariz, M.R. 2000, A\&A 354, 193

Hillier, D.J., Miller, D.L. 1998, ApJ 497, 407

Hubeny, I., Heap, S.R., Lanz, T. 1998, in: I.D. Howarth (ed.), Bouder-Munich II: Properties of Hot, Luminous Stars, ASP-CS 131, 108

Martins, F., Schaerer, D., Hillier, D.J. 2002, A\&A 382, 999

Pauldrach, A.W.A., Hoffmann, T.L., Lennon, M. 2001, A\&A 375, 161

Schaerer, D., Schmutz, W. 1994, A\&A 288, 231

Schmutz, W. 1998, in: I.D. Howarth (ed.), Bouder-Munich II: Properties of Hot, Luminous Stars, ASP-CS 131, 119

Vacca, W.D., Garmany, C.D., Shull, J.M. 1996, ApJ 460, 914 